\title{
ARTICLE \\ Developmental and behavioral effects in neonatal and adult mice following prenatal activation of endocannabinoid receptors by capsaicin
}

\author{
Alex Perchuk ${ }^{1}$, Sonya M. Bierbower ${ }^{1}$, Ana Canseco-Alba ${ }^{1}$, Zoila Mora ${ }^{1}$, Lauren Tyrell ${ }^{1}$, Neal Joshi ${ }^{1}$, Norman Schanz ${ }^{1}$, \\ Georgianna G. Gould ${ }^{2}$ and Emmanuel S. Onaivi ${ }^{1}$
}

Despite the apparent abundance of ligand-gated transient receptor potential vanilloid type 1 (TRPV1) and possible cross talk between the endocannabinoid and endovanilloid systems in the central nervous system (CNS), it is unclear what role TRPV1 receptor activation in CNS plays in neurobehavioral development. We previously reported that capsaicin or WIN55212-2 induces risk aversion in the plus-maze test, which was dependent on the gender and mouse strain used. In this study, pregnant BALBc mice were administered capsaicin ( 1.0 or $4.0 \mathrm{mg} / \mathrm{kg}$, i.p.) during the second week of gestation. Developmental effects of prenatal exposure to capsaicin were assessed in neonates, and behavioral effects were assessed in adult offspring. Gender- and dose-specific variations in ultrasonic vocalizations, weight gain, righting reflex, and general activity of the pups were observed. Prenatal exposure to capsaicin altered plus-maze performance, especially with further exogenous capsaicin challenge. Furthermore, dose- and gender-specific effects were evident in the conditioned place preference/aversion paradigm following conditioning with capsaicin in adult animals. The capsaicin-induced aversion in the plus-maze test was enhanced by WIN55212-2 and blocked by pretreatment with vanilloid antagonist capsazepine or the $\mathrm{CB}_{1}$ receptor antagonist rimonabant, demonstrating an interaction between the endocannabinoid and endovanilloid systems in CNS. Taken together, the interaction between the endocannabinoid and endovanilloid signaling systems can be exploited for therapeutic applications in health and disease.

Keywords: vanilloids; cannabinoids; conditioned place preference; prenatal exposure; plus-maze; reward; capsaicin, WIN55212-2; capsazepine; rimonabant

Acta Pharmacologica Sinica (2019) 40:418-424; https://doi.org/10.1038/s41401-018-0073-z

\section{INTRODUCTION}

In parallel with the remarkable advances in marijuana-cannabinoid research, groundbreaking work has led to deeper insights into the biological and behavioral effects of the activation of vanilloid receptors by capsaicin (Fig. 1) in chilli peppers. Furthermore, the mechanisms underlying the effects of capsaicin when consumed by humans and animals are being intensively investigated [1]. Similar to the way natural cannabinoids, such as $\Delta^{9}-$ THC in marijuana, activate endogenous cannabinoid receptors (CBRs) [2], a natural vanilloid capsaicin, the pungent chemical present in hot chili peppers, activates the cloned ligand-gated transient receptor potential vanilloid type 1 (TRPV1) protein, a nonselective cation channel with six transmembrane domains [3]. Like endocannabinoids, endogenous capsaicin-like substances called endovanilloid ligands, e.g. $\mathrm{N}$-arachidonoyldopamine and $\mathrm{N}$-oleoyldopamine, have been identified and shown to differentially activate both TRPV1 and $\mathrm{CB}_{1}$ Rs [4]. TRPV1 receptors are present in the brain and peripheral tissues [4]. There appear to be differences but also some similarities between the actions of cannabinoid and vanilloid agonists and antagonists. For instance, smoking marijuana or administration of cannabinoids can activate CBRs and
TRPV1 through endocannabinoids in the central nervous system (CNS), whereas capsaicin (in hot peppers) can also indirectly activate CBRs via cyclic AMP and directly activate TRPV1 by opening the ion channels. While cannabinoids mediate antinociception and antithermal effects centrally and peripherally through CBRs, capsaicin mediates nociception and thermal effects both centrally and peripherally through TRPV1. A possible interaction between the cannabinoid and vanilloid signaling systems has been suggested because of chemical similarity between synthetic agonists of vanilloid receptors, such as olvanil, and the endocannabinoid, anandamide (Fig. 1) [5]. Anandamide was the first endocannabinoid discovered [6], and it remains the most widely studied, yet this compound acts as only a partial agonist at CBRs. This, in turn, has led some to question how an endogenous natural ligand can act as a partial agonist of its own receptor [7]. It has been suggested that the physiological significance of anandamide may be as an endogenous ligand of a receptor other than CBRs or as a modulator of ion channels [7]. To date, the only well characterized, non-cannabinoid site of action for anandamide is at TRPV1, whereas the full agonist at CBRs is 2-arachidonyl glycerol. Therefore it is tempting to speculate that an interaction or cross

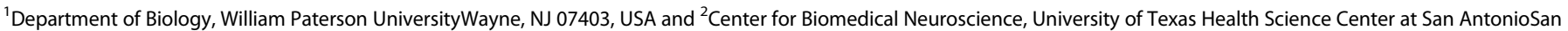
Antonio, TX 78229, USA

Correspondence: Emmanuel S. Onaivi (Onaivie@wpunj.edu)

Received: 12 January 2018 Revised: 12 June 2018 Accepted: 13 June 2018

Published online: 10 July 2018 

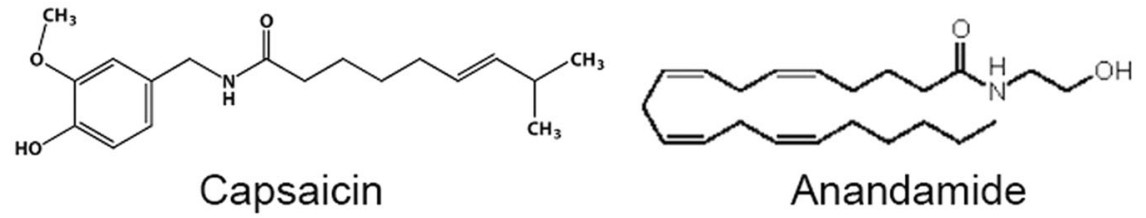

Fig. 1 Chemical structures of a typical endovanilloid capsaicin and an endocannabinoid anandamide

talk exists between the G-protein-coupled CBR system and the vanilloid system as demonstrated previously [8]. Prenatal exposure to cannabinoids has been shown to produce a reliable decrease in birth weight in animals [9], but this is the only postnatal effect on offspring that has been reliably documented. Studies examining long-term postnatal effects have generally been inconsistent. The evidence to date indicates that cannabinoids can affect gestational length and labor, and can induce maturational delays, and that these substances affect a myriad of physiological processes in the developing offspring, including effects on behavioral parameters in laboratory animals [10]. In light of the apparent abundance of TRPV 1 and possible cross talk between the endocannabinoid and endovanilloid systems in the CNS [11], this study was designed to investigate the developmental and behavioral effects of capsaicin-induced activation of these receptors in the early stages of embryological development. Neonatal neurobehavioral development was assessed using reflex tests as previously described [12]. Adult behavioral studies were conducted using the plus-maze and conditioned place preference paradigms.

\section{MATERIALS AND METHODS}

Animal and treatment

All experimental procedures followed the guidelines approved by the Institutional Animal Care and Use Committee of the William Paterson University of New Jersey in accordance with $\mathrm{NIH}$ guidelines for the care and use of laboratory animals. Virgin female BALBC mice weighing 18-20 g were housed from birth in individual cages in a climate-controlled colony room under a 12-h light-dark cycle (lights on at 8:00 a.m.) and constant temperature $\left(23 \pm 1^{\circ} \mathrm{C}\right)$. Animals were given ad libitum access to food and water, and each animal was handled daily. At adult age ( $>8$ weeks of life), females were allowed to stay with a male for mating, and those animals showing the presence of sperm as vaginal plugs were accepted as pregnant and used for the capsaicin exposure studies. The day on which sperm plugs were identified was designated the first day of gestation. A total of 54 pups were used. They were split into 9 groups for the conditioned place preference/aversion (CPP/CPA) and plusmaze tests ( $n=6 /$ group).

\section{Prenatal drug exposure}

Pregnant females received an intraperitoneal (i.p.) injection of either $1.0 \mathrm{mg} / \mathrm{kg}$ or $4.0 \mathrm{mg} / \mathrm{kg}$ capsaicin on gestation days 7,9 , 11 , and 13 for a total of four treatments (Fig. 2). The doses were chosen based on the dose-response pilot studies carried out by Onaivi et al. (unpublished data). Capsaicin (8-methyl- $N$ vanillyl-6-nonenamide) was dissolved in emulphor:95\% EtOH: $\mathrm{H}_{2} \mathrm{O}$ in a 1:1:18 ratio. The solution without the drug was used as a vehicle for control injections. All pregnant females were checked for births daily at 11 a.m. beginning on gestation day 17 , as the normal gestation period for BALBC strain of mice is between 19 and 21 days. The day when the births were observed was designated as postnatal day (PND) zero. All newborn pups were toe-clipped for identification purposes on PND 2, and developmental studies were carried out between PND 3 and PND 11.
Developmental studies in neonates

Ultrasonic vocalization (USV) in pups was assessed daily using a Mini-3 bat detector (Ultra Sound Advice, London UK) at $70 \mathrm{~Hz}$. Each pup was removed from the nest and placed into a cottonpadded plastic cup for $5 \mathrm{~min}$ at room temperature. Subsequently, for each pup, the number of calls during $30 \mathrm{~s}$ was counted, and the litter averages were plotted for male and female pups. The data are presented in the form of USVs/min (means \pm SEM). The pups were also weighed and assessed for general activity in an open field, a $24 \times 24 \mathrm{~cm}$ box divided into nine $8 \times 8 \mathrm{~cm}$ squares. The pup was considered to have traversed a square when all of its paws moved onto the adjacent square. The data are presented as the means of litter averages \pm SEM. The following reflex tests were also used on days 5, 8, and 11 as described by Fox [12]: negative geotaxis-when the subject was placed on a $45^{\circ}$ slope with its head pointing down the incline, it had $15 \mathrm{~s}$ to turn around and crawl up the slope; righting reflex-when the animal was placed on its side, it was expected to immediately turn over to rest in the normal position with all four paws on the ground; motor performance in pups was assessed with the use of a rota-rod at 5 rounds/min - the amount of time that the animal was able to remain on the rota-rod was measured, up to a maximum of $15 \mathrm{~s}$ (data are not shown). Following weaning on PND 21, pups were separated by sex and litter and maintained at 2-4 animals to a cage. Animals housed alone were excluded from further behavioral testing.

Behavioral studies in adult mice

CPP/CPA. The behavioral studies on the adult animals began on PND 50. The CPP/CPA induced by capsaicin was investigated. The conditioning apparatus consisted of two compartments separated by a guillotine door, one compartment with a mesh floor and the other with rails of $3 \mathrm{~mm}$ in diameter. During phase $\mathrm{I}$, the preconditioning period, mice received a priming capsaicin injection in their home cage on day 1 to counteract the possible dysphoric consequences of the first drug exposure as previously described [13]. Subsequently, the animals were allowed to explore the two compartments of the shuttle box for $15 \mathrm{~min} /$ day for the following two days. To check for any initial unconditioned preference for either of the two sides, the time spent by each animal in each compartment was recorded on the second day. In the conditioning period (phase II), mice from each prenatal treatment group were further subdivided into three experimental sets, receiving either 0.5 or $1.0 \mathrm{mg} / \mathrm{kg}$ capsaicin or a vehicle. The conditioning phase consisted of pairing of one distinctive compartment with capsaicin, alternated with two pairings of the other compartment with the vehicle. Animals in each drug group were randomly assigned to compartments and injection orders, both of which were counterbalanced. Each animal was placed in the appropriate compartment for $10 \mathrm{~min}$ after receiving an i.p. injection of the drug or vehicle. The guillotine door was closed to confine the animal to the compartment for $15 \mathrm{~min}$. In the testing phase, no drug or vehicle was injected, and the guillotine door was raised to allow the animal access to either chamber. Mice were placed in the middle and allowed to move about freely for $20 \mathrm{~min}$. The amount of time spent in each compartment was recorded. Since all animals tested showed a significant initial preference 

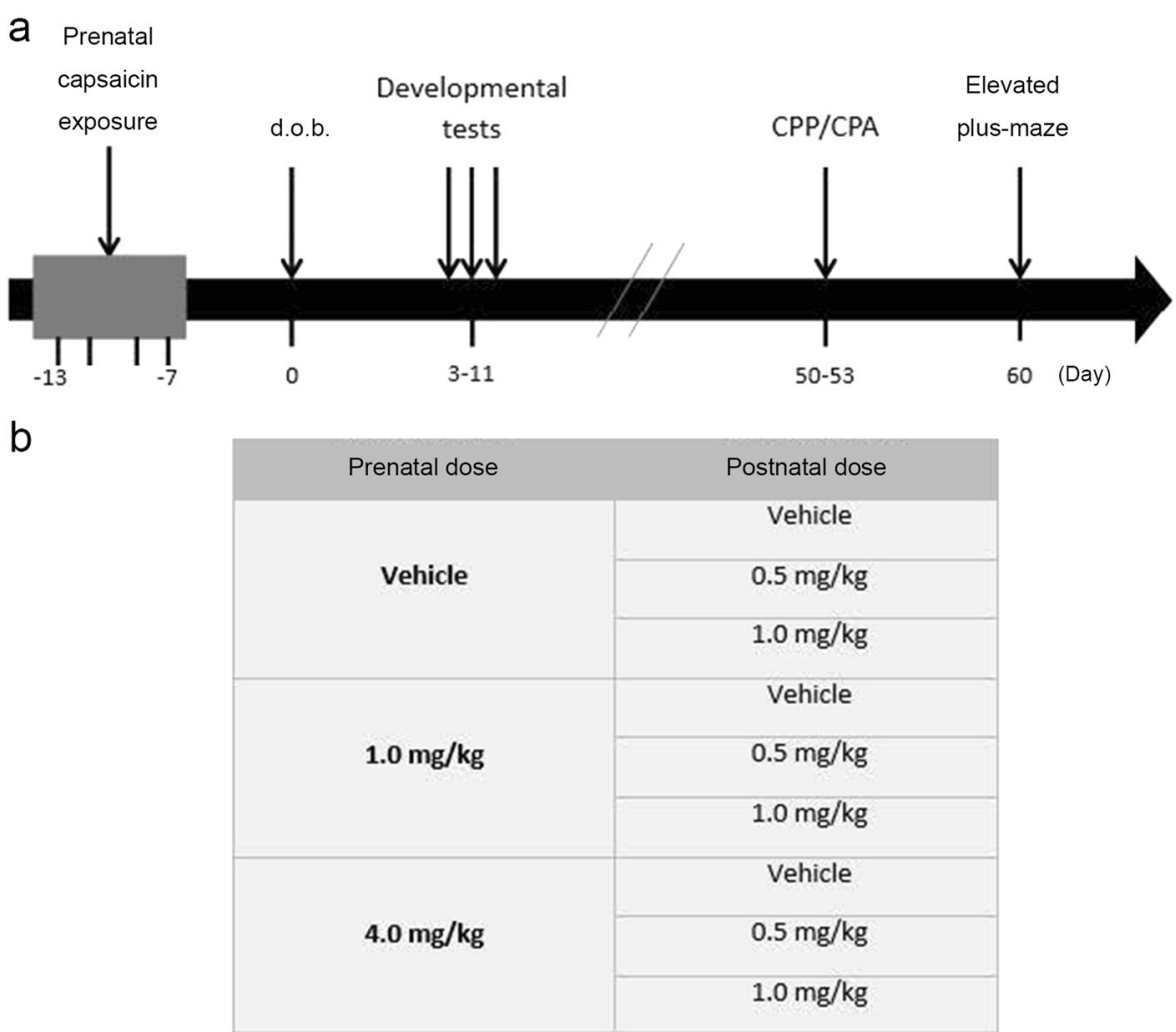

Fig. 2 Experimental design and timeline. a Prenatal capsaicin doses are represented as four doses on gestational days 7-13. The d.o.b. represents postnatal day (PND) 0 followed by PNDs 3-11 when developmental tests were performed. The CPP/CPA test was performed on PNDs 50-53 followed by a 1-week washout period, and on PND 60, the elevated plus-maze test was performed. b The experimental groups used for the CPP/CPA and elevated plus-maze tests ( $n=6 /$ group)

for the square mesh compartment of the shuttle box during the preconditioning phase (phase I), a biased approach was used in analyzing the data. That is, those animals that received a capsaicin injection on the mesh side of the shuttle box were used to establish the conditioned place aversion (if present), and the animals that received the capsaicin injection on the initially un-preferred, rail side of the shuttle box were evaluated for the conditioned place preference.

Elevated plus-maze test. Following a 1-week washout period, female mice were tested in the elevated plus-maze following an acute challenge with either 0.5 or $1.0 \mathrm{mg} / \mathrm{kg}$ capsaicin or a vehicle injection. The elevated plus-maze consisted of two open arms and two enclosed horizontal perpendicular arms $50 \mathrm{~cm}$ above the floor, similar to the apparatus we previously described [14]. The arms were linked by a central platform and arranged in a "plus sign" (+). The apparatus was made of dark vinyl Plexiglas material and mounted on a clear plastic base. The plus-maze was automated using pairs of photocells with their appropriate receivers strategically located to enable computer (IBM PC) monitoring of animal movement and location to measure anxiety-like behavior. The junction of the four arms formed a square central platform $(5 \times 5 \mathrm{~cm})$. The test began with the animal being placed in the center of the apparatus facing one of the enclosed arms, and the animal was then allowed to explore freely for $5 \mathrm{~min}$. The total time spent in the closed and open arms and the numbers of entries for each compartment were recorded by the computerized photo-beam system. The number of entries was automatically recorded when a subset of the photocell beams in the open and closed arms and the diagonal medians of the central platform were interrupted. With this arrangement, the movement and location of the animals during the 5-min test were continuously displayed, monitored, and recorded. Testing was initiated $10 \mathrm{~min}$ after vehicle or capsaicin.

Statistical analysis

The data were analyzed using GraphPad Prism (GraphPad Software Inc., San Diego, CA, USA). Significant differences between the treatment groups were identified by one-way ANOVA followed by Bonferroni's test. Dunnett's $t$-test for multiple comparisons was performed where appropriate. Differences were considered significant at the a priori level of $P<0.05$.

\section{RESULTS}

Developmental studies in neonates: USVs

Figure 3 summarizes the results of the USV rates in male (Fig. 3 a) and female (Fig. $3 \mathrm{~b}$ ) pups. In the control males (CM) group (vehicle treated), the rate of USV peaked on day 5 (34.8 \pm 10.8$)$; in the group $M 1(1.0 \mathrm{mg} / \mathrm{kg}$ capsaicin), the peak rate was observed between PND 3 and $4(24.1 \pm 3.4$ and $23.0 \pm 5.3)$; and in the group M4 $(4.0 \mathrm{mg} / \mathrm{kg}$ capsaicin), the USV rate was highest on day 6 $(46.0 \pm 18.4)$ (Fig. 3a). The only significant difference in the call rate in males was observed on PND 9, when the calling rate of the pups exposed to $4.0 \mathrm{mg} / \mathrm{kg}$ capsaicin in utero was significantly greater than that in control subjects $(P<0.05)$. In females, group CF showed the highest USV rate on day 5 (33.6 \pm 7.2$)$, whereas groups F1 and F4 had peaks on day 4 (31.4 \pm 10.6 and $39.6 \pm 13.2$, respectively) (Fig. $3 b$ ). The only significant difference was an increase in call rates on PND 8 in female pups exposed to $4.0 \mathrm{mg} /$ $\mathrm{kg}$ capsaicin in utero compared to controls $(P<0.05)$. 
Developmental studies in neonates: general activity and weight changes

Prenatally exposed mice in group I were exposed to vehicle, mice in group II were exposed to $1.0 \mathrm{mg} / \mathrm{kg}$ capsaicin, and mice in group III were exposed to $4.0 \mathrm{mg} / \mathrm{kg}$ capsaicin. General activity was increased in group II male pups on PND 11 compared to the other groups $(P<0.001)$; an increase in activity was also observed in group III pups on day 11 compared to controls $(P<0.001)$ (Fig. 4a). No significant changes in general activity were observed in age-matched female pups (Fig. 4b). No significant performance differences in the tests of negative geotaxis, rota-rod performance, or righting reflex were noted among the groups (data not shown). The results of changes in weight gain are summarized in Fig. 4c, d for males and females, respectively. Compared to the controls, there was a significant dose-dependent delay in weight gain on PNDs 5, 8, and 11 in male pups prenatally exposed to either 1.0 or $4.0 \mathrm{mg} / \mathrm{kg}$ capsaicin ( $P<0.01$ ) (Fig. 4c). The only significant weight gain delay in females was observed on PND 5 in pups prenatally exposed to $4.0 \mathrm{mg} / \mathrm{kg}$ capsaicin $(P<0.01)$ (Fig. $4 \mathrm{~d}$ )
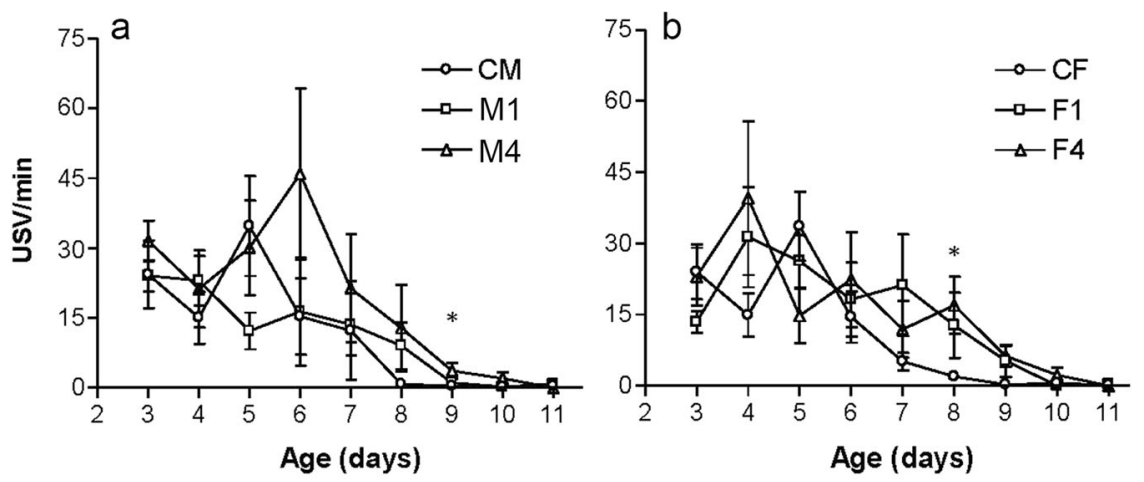

Fig. 3 The ultrasonic vocalization profile for male (a) and female (b) BALBc mouse pups on postnatal days (PNDs) $3-11$. Means \pm SEM. ${ }^{*} P<0.05$ vs. control. $n=9$
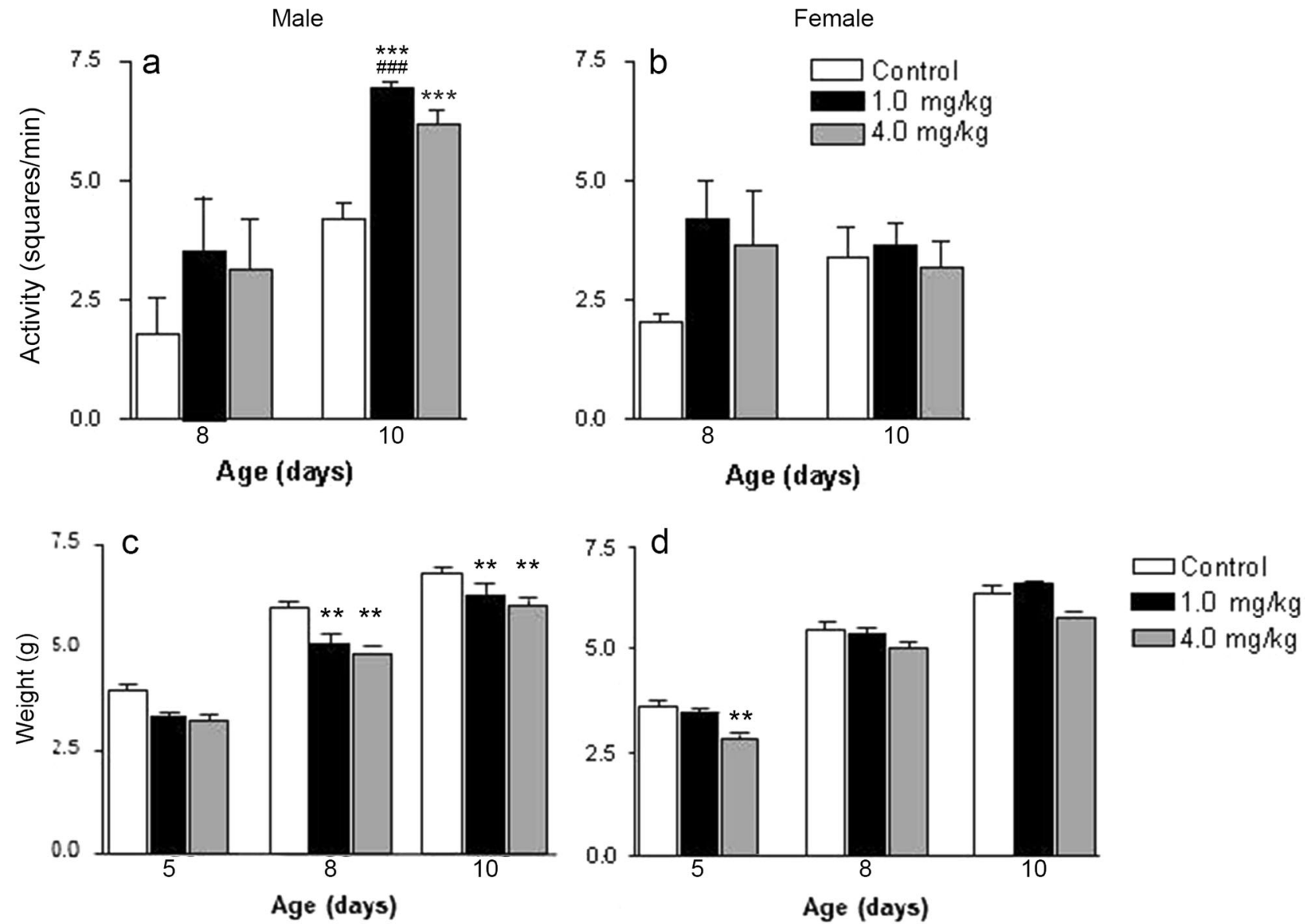

Fig. 4 General activity $(\mathbf{a}, \mathbf{b})$ and weight gain $(\mathbf{c}, \mathbf{d})$ profiles for male and female BALBc mouse pups prenatally exposed to either 1.0 or 4.0 mg/ $\mathrm{kg}$ capsaicin or vehicle. Means \pm SEM. ${ }^{* *} P<0.01,{ }^{* * *} P<0.001$ vs. control; ${ }^{\# \#} P<0.001$ vs. $4.0 \mathrm{mg} / \mathrm{kg}$ group. $n=9$ 
Behavioral studies in adult offspring prenatally exposed to capsaicin

$C P P / C P A$. The results are summarized in Table 1 . Animals showed no preference for the rail or mesh compartments. Conditioning with capsaicin at either 0.5 or $1.0 \mathrm{mg} / \mathrm{kg}$ for two alternate days in the rail compartment of the CPP shuttle box resulted in a significant $(P<0.05)$ increase in the amount of time an animal spent in the compartment, comparing the drug between naïve and prenatally drug-exposed animals treated with vehicle at the conditioning phase. There were no sex-specific differences observed in the conditioning paradigm; therefore, the data for sexes were pooled.
Elevated plus-maze. The total time spent in the enclosed arms in the group prenatally exposed to $4.0 \mathrm{mg} / \mathrm{kg}$ capsaicin with vehicle challenge was $113.2 \pm 24.5 \mathrm{~s}$, and upon acute challenge with capsaicin, it was $220.5 \pm 22.5$ and $230.6 \pm 30.4 \mathrm{~s}$ for 0.5 and $1.0 \mathrm{mg} / \mathrm{kg}$ capsaicin, respectively $(P<0.05$ vs. vehicle for both conditions, Fig. 5a). No significant differences based on any other plus-maze parameters were observed. However, in naïve mice not prenatally exposed to capsaicin, treatment with the $C_{1}$ and $C B_{2}$ agonist WIN55212-2 induced in mice aversion to the open arm of the plus-maze in mice. The aversive behavior induced in mice following treatment with capsaicin, which was dependent on gender and strain, was enhanced by pretreatment with

Table 1. Capsaicin-induced CPP in BALBc mice prenatally exposed to either 1.0 or $4.0 \mathrm{mg} / \mathrm{kg}$ capsaicin

\begin{tabular}{|c|c|c|c|c|c|c|c|c|c|c|}
\hline \multirow{3}{*}{ Prenatal } & \multicolumn{10}{|c|}{ Postnatal } \\
\hline & \multicolumn{4}{|c|}{ Vehicle } & \multicolumn{3}{|c|}{ Capsaicin $0.5 \mathrm{mg} / \mathrm{kg}$} & \multicolumn{3}{|c|}{ Capsaicin $1.0 \mathrm{mg} / \mathrm{kg}$} \\
\hline & Before & After & Mean diff & $P$ & After & Mean diff & $P$ & After & Mean diff & $P$ \\
\hline Vehicle & 477 & 496 & 19 & 0.6730 & 601 & 124 & $0.0034^{*}$ & 573 & 96 & $0.0304^{*}$ \\
\hline $1 \mathrm{mg} / \mathrm{kg}$ & 527 & 493 & -34 & 0.0953 & 672 & 145 & $0.0377^{*}$ & 611 & 84 & $0.0339^{*}$ \\
\hline $4 \mathrm{mg} / \mathrm{kg}$ & 492 & 511 & 19 & 0.2740 & 597 & 105 & $0.0463^{*}$ & 537 & 45 & $0.0248^{*}$ \\
\hline
\end{tabular}
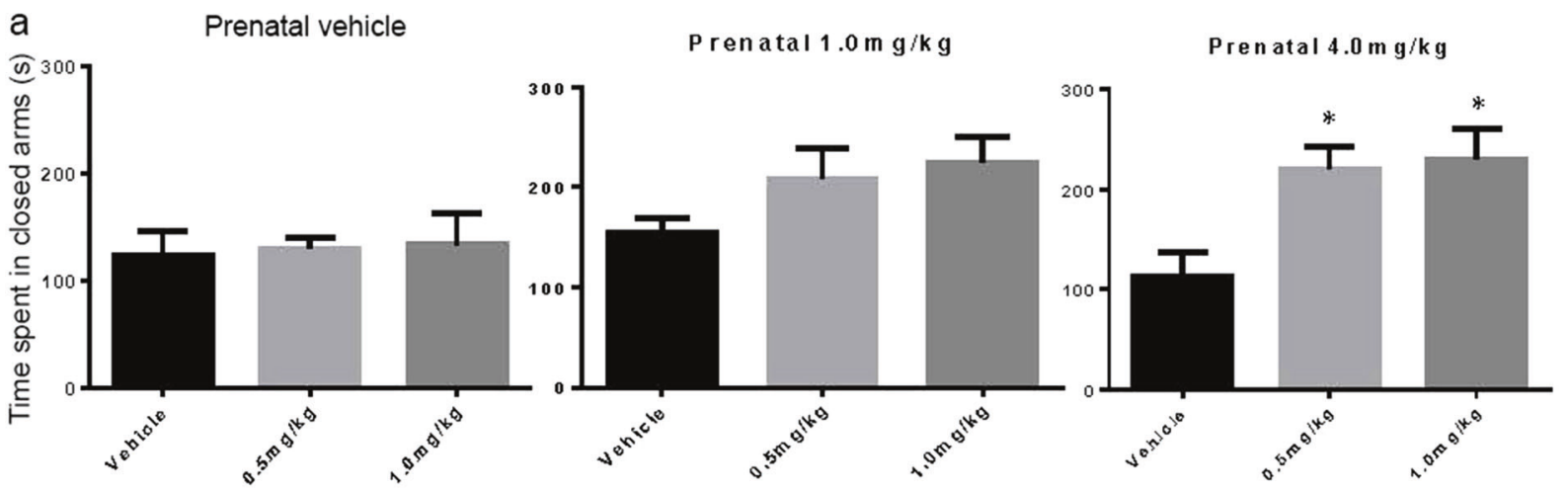

Postnatal challenge doses (capsaicin)

b

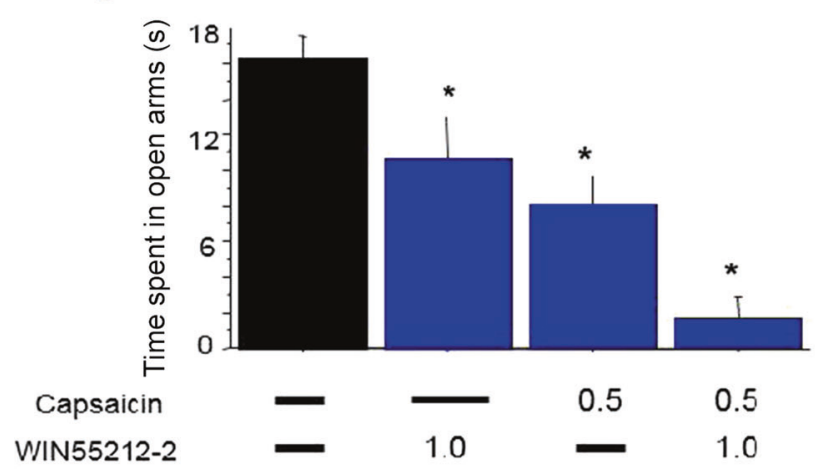

C
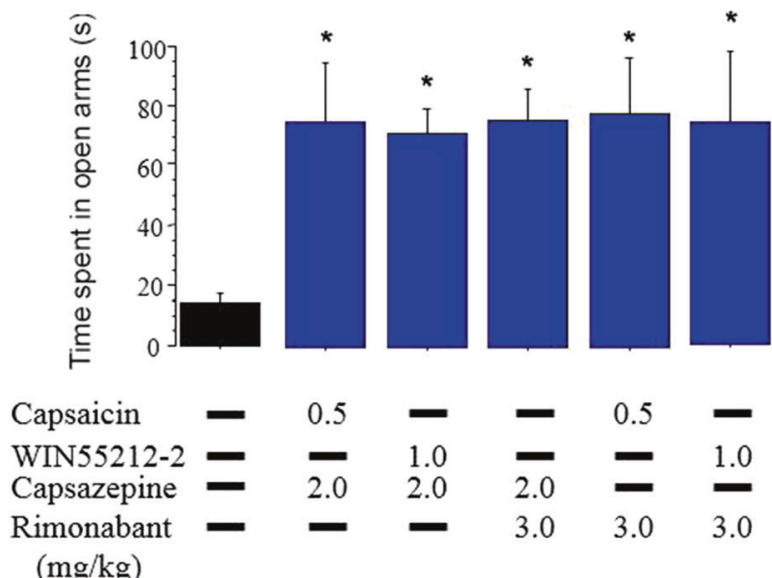

Fig. 5 Time spent in closed (a) and open (b, c) arms of the elevated plus-maze (only component to reach significance, other data not shown). b The effect of pretreatment with capsazepine or rimonabant 30 min prior to the administration of capsaicin or WIN55212-2 on mouse aversive behavior in the plus-maze test. c The acute effect of selected doses of WIN55212-2 alone, or capsaicin alone, or in combination on the performance of mice in the plus-maze test. For the combination, mice received WIN55212-2 at 20 min prior to the administration of capsaicin, and were then tested in the maze $10 \mathrm{~min}$ after the administration of capsaicin. Means $\pm \mathrm{SEM}$ ( $n=10$ mice per group). ${ }^{*} P<0.05$ vs. vehicle; ANOVA with Dunnett's multiple comparison tests 
WIN55212-2 (Fig. 5b). The vanilloid antagonist capsazepine and the $\mathrm{CB}_{1} \mathrm{R}$ antagonist rimonabant blocked the aversions induced by WIN55212-2 and capsaicin (Fig. 5c), indicating cross talk between the cannabinoid and vanilloid systems.

\section{DISCUSSION}

In the present study, our results demonstrate that prenatal exposure to capsaicin, the pungent chemical in hot chilli peppers, produced gender-dependent developmental effects in neonates and adult offspring. In the pups, gender- and dosespecific variations in USVs, weight gain, righting reflex, and increased general activity of the male pups were recorded. USVs emitted by altricial rodent pups are whistle-like sounds characterized by frequencies ranging between 30 and $90 \mathrm{kHz}$ [15]. It has been further suggested that the USV profile can be used to assess neurobehavioral development in infant rodents [15]. For instance, the late peak in the USV rate may indicate slow development, while an early peak may be characteristic of a developmental acceleration. Furthermore, several studies have also been conducted to investigate stress-induced vocalizations as a model for studying anxiety [16]. To make this measurement even more attractive to researchers, the ontogenetic profile of the age-related rates of USVs is highly strain-specific. For instance, it has been reported [17] that the BALBc strain has a peak ultrasound rate on PND 3. However, a peak USV rate between PNDs 3 and 6 is considered a sign of normal development. Although it certainly appears that prenatal capsaicin exposure had some variable gender- and dosespecific effects, none of the USV profiles in this study appeared to indicate that prenatal exposure to capsaicin at either 1.0 or $4.0 \mathrm{mg} / \mathrm{kg}$ has any adverse neurodevelopmental effects. Further investigation is necessary to determine whether the variation in the USV rate is related to possible anxiolytic or anxiogenic effects that prenatal exposure to capsaicin may have on animals' development. On the other hand, the observed variation may be due to drug-independent natural variability. Prenatal exposure to capsaicin appeared to have a more significant effect on weight gain in male pups than in females. While male pups exposed to either 1.0 or $4.0 \mathrm{mg} / \mathrm{kg}$ capsaicin in utero appeared to be adversely affected, possibly due to inactivation of a neural circuit known as the gut-brain axis by capsaicin, as reported [18] or with a decrease in milk consumption by pups due to capsaicin residue present in maternal milk, the reason for such sex specificity is unclear.

Conditioned place preference is a behavioral model used to measure the rewarding properties induced by the administration of a drug. In this paradigm, the rewarding properties of a compound are associated with the particular characteristics of a given environment. After conditioning, the animal will prefer to spend more time in the environment associated with the drug. A similar place-conditioning animal model can also be used to explore the aversive properties of a drug. In this case, the animal will avoid staying in the compartment associated with a compound producing aversive effects. The administration of cannabinoid agonists produces dose-dependent variable preference- or aversion-like responses in the place-conditioning paradigm [13]. A significant strain specificity suggesting a lack of genetic uniformity in endocannabinoid systems between different mouse strains has also been reported [19]. Although capsaicin has been used by humans throughout recorded history, its rewarding properties in the CPP/CPA paradigm have not been systematically evaluated. One objective of this study was to determine whether further exogenous challenge with capsaicin $(0.5$ or $1.0 \mathrm{mg} / \mathrm{kg}$, i.p.) in prenatally exposed animals would produce a CPP in mice drugpaired to their less-preferred compartment, or an aversion in another group of mice that were drug-paired to their more preferred compartment. This allowed us to determine whether prenatal exposure to capsaicin is a factor in drug reward. The performance of the animals in the CPP/CPA paradigm indicates an inconsistent profile of the action of capsaicin in the reward pathway (if such a pathway exists). The data obtained in this study appear to be in line with the results obtained in cannabinoid studies, in which CPP/CPA has been difficult to establish. In an earlier pilot study carried out by Onaivi and Perchuk (unpublished data), it was observed that while conditioning with $1.0 \mathrm{mg} / \mathrm{kg}$ capsaicin induces conditioned place aversion to the drug-paired compartment, conditioning with $0.5 \mathrm{mg} / \mathrm{kg}$ capsaicin had no significant effect. In the present experiment, conditioning with either drug dose appeared to induce dose- and treatmentindependent place preference when capsaicin was paired with the initially un-preferred compartment, while no preference or aversion was present when capsaicin was paired with the initially preferred compartment, a typical finding in a biased conditioning set up. In the present model, the behavioral effects of capsaicin may be attributable to the release of endovanilloids and/or endocannabinoids, which are known to modulate both TRPV1 and CB receptors. However, the direct physiological and behavioral effects of capsaicin in this paradigm may be better studied using TRPV1 knockout animals, or using capsazepine, a vanilloid antagonist, in pharmacological studies. These genetic and pharmacological approaches may enhance our understanding of the rewarding mechanisms associated with capsaicin treatment.

The plus-maze used in this study was similar to that validated and described previously [20], and has been widely used in a number of test conditions studying anxiolytic or anxiogenic effects of various compounds. In the plus-maze test, the prenatal exposure to capsaicin enhanced the behavioral aversion of mice to the open arms with exogenous capsaicin challenge (independent of the dose) in comparison to controls. This may indicate that down-regulation of TRPV1 in response to prenatal vanilloid exposure may play a significant role in anxiogenesis. Prenatal exposure to capsaicin, either 1.0 or $4.0 \mathrm{mg} / \mathrm{kg}$, had dose- and gender-specific effects on the development of mouse pups. Although it is difficult to attribute these variations exclusively to the physiological and pharmacological action of capsaicin without further molecular testing, it would appear reasonable to conclude that in utero exposure to capsaicin induces discrete developmental changes in the mouse model. Thus, the nature of the cross talk between the endocannabinoid and endovanilloid systems (if involved in the action of capsaicin) remains to be established. The aversions induced by capsaicin in the plus-maze test were enhanced by WIN55212-2 and blocked by capsazepine or rimonabant, indicating an interaction between the endocannabinoid and endovanilloid systems. Overall, the interaction between the endocannabinoid and endovanilloid signaling systems can be exploited for therapeutic applications in health and disease. Hence, future studies are needed to elucidate the effects of capsaicin at the cellular and molecular levels.

\section{ACKNOWLEDGEMENTS}

$A P, S M B, A C-A, Z M, L T, N S, G G G$, and ESO are supported by William Paterson University. AC-A is also currently supported by the Mexican National Council of Science and Technology (CONACYT \# CVU332502/232728). ESO was supported by NIH grant DA032890. GGG is now at the University of Texas Health Science Center in San Antonio and is supported by grants from DoD \#AR110109 as well as by the Morrison Trust, and Lindow, Stephens and Treat, LLP. This is dedicated to the memory of the sudden tragic death of our co-author NS.

\section{AUTHOR CONTRIBUTIONS}

ESO conceptualized the study and wrote the manuscript. AP, SMB, AC-A, ZM, LT, NS, GGG, and ESO contributed expertise and support in performing the experiments. NJ 
revised the figures and NJ and GGG revised the manuscript. All authors approved the publication of this manuscript.

\section{ADDITIONAL INFORMATION}

Competing interests: The authors declare that they have no conflict of interest.

Publisher's note: Springer Nature remains neutral with regard to jurisdictional claims in published maps and institutional affiliations.

\section{REFERENCES}

1. Sharma SK, Vij AS, Sharma M. Mechanisms and clinical uses of capsaicin. Eur J Pharmacol. 2013;720:55-62.

2. Onaivi ES, Ishiguro $H, G u S$, Liu QR. CNS effects of CB2 cannabinoid receptors: beyond neuro-immuno-cannabinoid activity. J Psychopharmacol. 2012;26:92-103.

3. Tominaga M, Julius D. Capsaicin receptor in the pain pathway. Jpn J Pharmacol. 2000;83:20-4.

4. Huang SM, Bisogno T, Trevisani M, Al-Hayani A, De Petrocellis L, Fezza F, et al. An endogenous capsaicin-like substance with high potency at recombinant and native vanilloid VR1 receptors. Proc Natl Acad Sci USA. 2002;99:8400-5.

5. Di Marzo V, Bisogno T, Melck D, Ross R, Brockie H, Stevenson L, et al. Interactions between synthetic vanilloids and the endogenous cannabinoid system. FEBS Lett. 1998;436:449-54.

6. Devane WA, Hanus L, Breuer A, Pertwee RG, Stevenson LA, Griffin G, et al. Isolation and structure of a brain constituent that binds to the cannabinoid receptor. Science. 1992;258:1946-9.

7. Sugiura T, Kondo S, Sukagawa A, Nakane S, Shinoda A, Itoh K, et al. 2-Arachidonoylglycerol: a possible endogenous cannabinoid ligand in brain. Biochem Biophys Res Commun. 1995;215:89-97.
8. Onaivi ES. An endocannabinoid hypothesis of drug reward and drug addiction. Ann NY Acad Sci. 2008;1139:412-21.

9. Abel EL. Effects of prenatal exposure to cannabinoids. NIDA Res Monogr. 1985;9:20-35

10. Dalterio SL. Cannabinoid exposure: effects on development. Neurobehav Toxicol Teratol. 1986;8:45-52.

11. Onaivi ES, Colas D, Hall FS, Taylor RE, Uhl GR, Akinshola BE. Interaction of cannabinoids and vanilloids: a biological basis of why some like it hot and others do not. Sixth IBRO World Congr Neurosci Abstr. 2003;2262:206.

12. Fox WM. Reflex-ontogeny and behavioral development of the mouse. Anim Behav. 1965;13:234-41.

13. Valjent $E$, Maldonado R. A behavioral model to reveal place preference to $\Delta^{9}$ tetrahydrocannabinol in mice. Psychopharmacology. 2000;147:436-8.

14. Onaivi ES, Bishop-Robinson C, Darmani NA, Sanders-Bush E. Behavioral effects of (+/-)-1-(2,5-dimethoxy-4-iodophenyl)-2-aminopropane, (DOI) in the elevated plus-maze test. Life Sci. 1995;57:2455-66.

15. Branchi I, Santucci D, Alleva E. Ultrasonic vocalization emitted by infant rodents: a tool for assessment of neurobehavioral development. Behav Brain Res. 2001;125:49-56.

16. Hahn ME, Schanz N. The effects of cold, rotation, and genotype on the production of ultrasonic calls in infant mice. Behav Genet. 2002;32:267-73.

17. Bell RW, Nitschke W, Zachman TA. Ultra-sounds in three inbred strains of young mice. Behav Biol. 1972;7:805-14.

18. Laskiewicz J, Królczyk G, Zurowski D, Enck P, Thor PJ. Capsaicin induced deafferentation enhances the effect of electrical vagal nerve stimulation on food intake and body mass. J Physiol Pharmacol. 2004;55:155-63.

19. Onaivi ES, Chakrabarti A, Gwebu E, Chaudhuri G. Neurobehavioral effects of $\Delta^{9}$ THC and cannabinoid (CB1) receptor gene statement in mice. Behav Brain Res. 1996;72:115-25.

20. Onaivi ES, Green MR, Martin BR. Pharmacological characterization of cannabinoids in the elevated plus maze. J Pharmacol Exp Ther. 1990;253:1002-9. 